\title{
A variational approach for dissipative quantum transport in a wide parameter space
}

\author{
Yu Zhang, ${ }^{1, \text { a) }}$ ChiYung Yam, ${ }^{1,2}$ YanHo Kwok, ${ }^{1}$ and GuanHua Chen ${ }^{1, b)}$ \\ ${ }^{1}$ Department of Chemistry, The University of Hong Kong, Pokfluam Road, Hong Kong \\ ${ }^{2}$ Beijing Computational Science Research Center, Beijing 100094, China
}

(Received 3 June 2015; accepted 31 August 2015; published online 14 September 2015)

\begin{abstract}
Recent development of theoretical method for dissipative quantum transport has achieved notable progresses in the weak or strong electron-phonon coupling regime. However, a generalized theory for dissipative quantum transport in a wide parameter space had not been established. In this work, a variational polaron theory for dissipative quantum transport in a wide range of electron-phonon coupling is developed. The optimal polaron transformation is determined by the optimization of the Feynman-Bogoliubov upper bound of free energy. The free energy minimization ends up with an optimal mean-field Hamiltonian and a minimal interaction Hamiltonian. Hence, second-order perturbation can be applied to the transformed system, resulting in an accurate and efficient method for the treatment of dissipative quantum transport with different electron-phonon coupling strength. Numerical benchmark calculation on a single site model coupled to one phonon mode is presented. @ 2015 AIP Publishing LLC. [http://dx.doi.org/10.1063/1.4930847]
\end{abstract}

\section{INTRODUCTION}

Quantum transport and energy dissipation in nanostructures have been of great interest, which requires the study and understanding of nonequilibrium phenomena of open quantum systems. ${ }^{1-7}$ In many previous studies on dissipative quantum transport, the coupling between the system and the phonon bath is considered as small. In these cases, secondorder perturbation (2PT) was employed, leading to a set of equations of motion (EOMs). ${ }^{6}$ However, there exist many open quantum systems with strong electron-phonon coupling. For instance, the coupling between system and bath in energy transfer process in photosynthetic complexes is comparable to the electronic coupling, ${ }^{8-10}$ strong electron-phonon coupling is also observed in resonant tunneling in molecular transport junctions. ${ }^{11,12}$ Even though there are several non-perturbative methods which can exactly evaluate the dynamics, such as hierarchical equations of motion (HEOM) ${ }^{13,14}$ quasiadiabatic propagator path integral (QUAPI) ${ }^{15}$ and multiconfiguration time-dependent Hartree (MCTDH) approach, ${ }^{16-18}$ these methods are computational demanding and nontrivial to be implemented.

There is another approach applies to systems with strong electron-phonon coupling, which makes use of polaron transformation. A polaron transformed second-order master equation for open quantum system has been developed accordingly. ${ }^{19-22}$ The polaron theory has also been employed to study the quantum transport in the strong coupling regime, ${ }^{23-29}$ in which strong phonon blockade effect is observed. The

\footnotetext{
a)Electronic mail: zhy@yangtze.hku.hk. Present address: Center for Bioinspired Energy Science and Department of Chemistry, Northwestern University, Evanston, IL 60208, USA.

b)Electronic mail: ghc@everest.hku.hk
}

polaron transformation extends the validity of perturbative treatment of electron-phonon interaction to strong coupling regime, provided that the electronic couplings are small compared to the typical electron-phonon coupling and bath frequency. However, in weak coupling regime where polarons fail to follow the system dynamics, the polaron theory performs worse than the standard perturbative approaches.

In order to overcome the shortcoming of polaron transformation based approaches, the variational method has been applied as a generalization of polaron transformation. ${ }^{30-34}$ In contrast with full polaron transformation, the variational polaron approach searches for an optimal amount of transformation by employing variational principle, based on the minimization of the Feynman-Bogoliubov upper bound of free energy. ${ }^{33}$ The optimal transformation is determined by the properties of the bath and system-bath coupling. Thanks to the variational principle, the variational polaron method applies in both weak and strong coupling regimes and is able to capture the dynamic behaviour in a wide parameter space. Moreover, compared to the many-particle approaches, the computational effort of variational polaron method is much lower.

Inspired by the recently developed variational polaron master equation, ${ }^{30,31}$ variational polaron method for dissipative quantum transport is developed in this work. The manuscript is organized as follows. Sec. II introduces the variational polaron theory for dissipative quantum transport. Starting from the variational polaron transformation, the transformed Hamiltonian is divided into two parts: a mean-field part and an interacting part. Free energy optimization ends up with an optimal mean-field Hamiltonian and a perturbation part which 2PT is applied. The variational polaron theory for quantum transport is applied to study a single site model with different electron-phonon coupling strength, which is given in Sec. IV. Finally, we summarize this work in Sec. V. 


\section{VARIATIONAL POLARON THEORY FOR QUANTUM TRANSPORT}

\section{A. Model Hamiltonian}

The problem of interest is an open system sandwiched between two leads. The electrons can transfer from one lead to the other through the system driven by bias voltage. The electrons have the probability of being scattered inelastically by phonons when they transport through the system. The corresponding Hamiltonian reads

$$
H=H_{S}+\sum_{\alpha}\left[H_{\alpha}+H_{S \alpha}\right]+H_{B}+H_{S B},
$$

where $H_{S}$ is the Hamiltonian of the system; $H_{B}$ is the Hamiltonian of phonon bath; $H_{\alpha}$ is the Hamiltonian of lead $\alpha$. The lead serves as electron bath; $H_{S B}$ and $H_{S \alpha}$ are the Hamiltonians of system-phonon coupling and system-lead interaction, respectively. Expressions of those Hamiltonians are

$$
\begin{aligned}
H_{S} & =\sum_{n} \epsilon_{n} c_{n}^{\dagger} c_{n}, \\
H_{\alpha} & =\sum_{k_{\alpha}} \epsilon_{k_{\alpha}} c_{k_{\alpha}}^{\dagger} c_{k \alpha}, \\
H_{S \alpha} & =\sum_{n, k_{\alpha}}\left[V_{k_{\alpha} n} c_{k_{\alpha}}^{\dagger} c_{n}+\text { H.c. }\right], \\
H_{B} & =\sum_{q} \omega_{q} b_{q}^{\dagger} b_{q}, \\
H_{S B} & =\sum_{n q} g_{n q} c_{n}^{\dagger} c_{n}\left(b_{q}^{\dagger}+b_{q}\right),
\end{aligned}
$$

where $\epsilon_{n}$ denotes the single-particle energy of the electronic state $n$ in the device and $c_{n}^{\dagger}\left(c_{n}\right)$ is the corresponding creation (annihilation) operator. Similarly, $\epsilon_{k_{\alpha}}$ and $c_{k_{\alpha}}^{\dagger}\left(c_{k_{\alpha}}\right)$ represent the single-particle energy and creation (annihilation) operator of $k$ th electronic state in lead $\alpha$, respectively. $V_{k_{\alpha} n}$ is the system-lead coupling strength. The phonon creation and annihilation operators are represented by $b_{q}^{\dagger}$ and $b_{q} . \omega_{q}$ and $g_{n q}$ are the corresponding the phonon energy and electron-phonon coupling constant, respectively.

In the absence of electron-phonon coupling, an exact time-dependent quantum transport theory has been developed recently. ${ }^{35-41}$ In presence of electron-phonon coupling, electron has the probability of being scattered inelastically by phonon. Previous theoretical studies of dissipative quantum transport usually focus on either weak or strong electronphonon coupling. In the weak coupling regime, 2PT (or lowest order expansion) is widely employed. In the strong electron-phonon coupling limit, the electron is localized by phonon scattering, which is more suitable to be described by the polaron theory. In this manuscript, a generalized theory for dissipative quantum transport in a wide parameter space of electron-phonon coupling is developed by extending the polaron theory to its variational version.

\section{B. Variational polaron transformation}

Similar to the polaron theory, the variational polaron theory starts from a unitary transformation generated by the operator

$$
U=\exp \left[\sum_{n q} \lambda_{n q} c_{n}^{\dagger} c_{n}\left(b_{q}^{\dagger}-b_{q}\right)\right],
$$

where $\lambda_{n q}=f_{n q} / \omega_{q}$. The unitary operator displaces the phonon oscillation in the positive and negative directions. The parameter $f_{n q}$ determines the magnitude of the displacement for each mode. It is obvious that $f_{n q}=g_{n q}$ corresponds to the conventional polaron transformation, while $f_{n q}=0$ denotes no transformation.

After the unitary transformation, except $\bar{H}_{\alpha}$ and $\bar{H}_{B}$ formally remain unchanged, other parts of Hamiltonians become

$$
\begin{aligned}
\bar{H}_{S} & =\sum_{n}\left(\epsilon_{n}+R_{n}\right) c_{n}^{\dagger} c_{n}, \\
\bar{H}_{S \alpha} & =\sum_{n k_{\alpha}}\left[V_{k_{\alpha} n} c_{k_{\alpha}}^{\dagger} c_{n} X_{n}+\text { H.c. }\right], \\
\bar{H}_{S B} & =\sum_{n q} \bar{g}_{n q} c_{n}^{\dagger} c_{n}\left(b_{q}^{\dagger}+b_{q}\right),
\end{aligned}
$$

where $R_{n}=\lambda_{n q}\left(f_{n q}-2 g_{n q}\right)$ is the renormalization energy which describes the shift of single-particle energy of electronic states induced by electron-phonon interaction. $\bar{g}_{n q}=g_{n q}$ $-f_{n q}$ is defined as the reduced electron-phonon coupling strength. $\bar{g}_{n q}$ indicates that the electron-phonon coupling is reduced by the unitary transformation. The system-lead coupling is dressed by phonon displacement operator $X_{n}$, where $X_{n}$ is defined as

$$
X_{n}=\exp \left[-\sum_{q} \lambda_{n q}\left(b_{q}^{\dagger}-b_{q}\right)\right] .
$$

In short, phonon's influence on electrons is transferred into three terms by the variational polaron transformation: (1) the polaron shift energy $R_{n}$, which represents the energy renormalization effect due to electron-phonon interaction; (2) the dressed coupling between system and leads by the displacement operator $X_{n}$; (3) reduced electron-phonon coupling $\bar{g}_{n q}$, which is zero in the strong coupling limit and approaches $g_{n k}$ in the weak coupling limit. It should be noted that the phonon-induced electron-electron interaction is ignored in above transformation for simplicity. In principle, variational polaron transformation ends up with an effective electron-electron interaction, $\bar{H}_{e e}=\sum_{m \neq n} U_{m n} c_{m}^{\dagger} c_{m} c_{n}^{\dagger} c_{n}$, where $U_{m n}=\sum_{q} \frac{f_{m q}\left(f_{n q}-2 g_{n q}\right)}{\omega_{q}}$. However, the treatment of electron-electron interaction is nontrivial, which is the focus of researches on electron-electron correlation. In this manuscript, we focus on the electron-phonon interaction and electronelectron interaction is neglected in this work. In the following numerical application, one-site model is used where the phonon-induced electron-electron interaction naturally vanishes. For multiple sites, one can get rid of treating effective electron-electron interaction by considering the "local" phonon only, i.e., each site is coupled to its own phonon modes.

The variational transformation results in a modification of system-lead coupling, which is mediated by the phonon displacement operator. The displacement operator can be divided into two parts as $X_{n}=\left\langle X_{n}\right\rangle+B_{n}$, where $\left\langle X_{n}\right\rangle$ is the expectation value of operator $X_{n}$ and $B_{n} \equiv X_{n}-\left\langle X_{n}\right\rangle$ is defined as the fluctuation around $\left\langle X_{n}\right\rangle$. Assuming phonon is in 
thermal equilibrium, $\left\langle X_{n}\right\rangle$ can be written as

$$
\left\langle X_{n}\right\rangle=\exp \left[-\frac{1}{2} \sum_{q} \lambda_{n q}^{2} \operatorname{coth}\left(\beta \omega_{q} / 2\right)\right],
$$

where $\beta$ is the inverse temperature. Consequently, $H_{S \alpha}$ can be separated into two terms,

$$
\begin{aligned}
\bar{H}_{S \alpha}^{0} & =\sum_{n, k_{\alpha}}\left[V_{k_{\alpha} n}^{R} c_{k_{\alpha}}^{\dagger} c_{n}+\text { H.c. }\right] \\
\bar{H}_{S \alpha}^{I} & =\sum_{n, k_{\alpha}}\left[V_{k_{\alpha} n} c_{k_{\alpha}}^{\dagger} c_{n} B_{n}+\text { H.c. }\right]
\end{aligned}
$$

where $V_{k_{\alpha} n}^{R}=V_{k_{\alpha} n}\left\langle X_{n}\right\rangle$ is the renormalized system-lead coupling mediated by phonon. Thus, the total transformed Hamiltonian can also be grouped into two parts. One part is the mean-field (or non-interacting) part $\bar{H}_{0}$ and the other one is the interacting Hamiltonian $\bar{H}_{I}$, where $\bar{H}_{0}$ is defined as

$$
\bar{H}_{0}=\bar{H}_{S}+\sum_{\alpha}\left(\bar{H}_{S \alpha}^{0}+\bar{H}_{\alpha}\right)+\bar{H}_{B} \equiv \bar{H}_{e}+\bar{H}_{B}
$$

and $\bar{H}_{I}=\bar{H}_{S B}+\sum_{\alpha} \bar{H}_{S \alpha}^{I}$.

It is obvious that the polaron transformation $\left(f_{n q}=g_{n q}\right)$ makes the explicit electron-phonon interaction term $\bar{H}_{S B}$ vanished. In contrast, the variational polaron transformation employs $f_{n q}$ as parameters and the values of which are determined by the minimization of an upper bound of free energy. The minimization inherent to variational approach allows to minimize the effect of $\bar{H}_{I}$ and obtain an optimal mean-field Hamiltonian $\bar{H}_{0}$. Thus, given an electron-phonon coupling strength, the variational polaron transformation in principle can end up with a small $\bar{H}_{I}$. Consequently, 2PT can be employed to account the effect of $\bar{H}_{I}$. Because of the singleparticle nature of $\bar{H}_{0}$ and second-order perturbative treatment of $\bar{H}_{I}$, variational polaron theory significantly reduces the computational complexity compared to the many-particle approaches.

\section{Minimization of the upper bound of free energy}

In this work, the Feynman-Bogoliubov upper bound of free energy $\mathrm{y}^{33}$ is chosen to determine the optimal value of $f_{n q}$ for the variational transformation. The upper bound of free energy is given as

$$
F_{u}=-\frac{1}{\beta} \ln \left\{\operatorname{tr}\left[e^{-\beta \bar{H}_{0}}\right]\right\}+\left\langle\bar{H}_{I}\right\rangle_{\bar{H}_{0}},
$$

where $\left\langle\bar{H}_{I}\right\rangle_{\bar{H}_{0}}=\operatorname{Tr}\left[\bar{H}_{I} e^{-\beta \bar{H}_{0}}\right] . F_{u}$ gives the upper bound of the true Free energy $F$, which is related to $F$ by the inequality $F \leq F_{u}$. It is obvious that the second term on the right hand side (RHS) of Eq. (9) is zero by construction. Besides, because $\left[\bar{H}_{B}, \bar{H}_{e}\right]=0, F_{u}$ can be further simplified and divided into two parts,

$$
F_{u}=F_{\mathrm{B}}-\frac{1}{\beta} \ln \left\{\operatorname{tr}\left[e^{-\beta \bar{H}_{e}}\right]\right\},
$$

where $F_{B}$ is the free energy of phonon bath. Because $F_{B}$ is not a function of $f_{n q}$, it is neglected in the minimization procedure. There are two ways to minimize the upper bound of free energy with respect to $f_{n q}$. One way is to treat the system and leads as a whole system and the upper bound of free energy can be numerically calculated by the diagonalization of $\bar{H}_{e}$. The other approach is to employ the linked cluster expansion method. ${ }^{42}$

The mean-field part of the transformed Hamiltonian, $\bar{H}_{0}$, is a function of the phonon-induced renormalization parameters $\left\{R_{n},\left\langle X_{n}\right\rangle\right\}$. Consequently, the Feynman-Bogoliubov upper bound of free energy $F_{u}$ can be minimized with respect to $\left\{R_{n},\left\langle X_{n}\right\rangle\right\}$. Hence, the minimization condition can be written as

$$
\frac{d F_{u}}{d f_{n q}}=\frac{\partial F_{u}}{\partial R_{n}} \frac{\partial R_{n}}{\partial f_{n q}}+\frac{\partial F_{u}}{\partial\left\langle X_{n}\right\rangle} \frac{\partial\left\langle X_{n}\right\rangle}{\partial f_{n q}}=0 .
$$

Using the expressions for $\left\{R_{n},\left\langle X_{n}\right\rangle\right\}$, we can evaluate the derivatives of $\left\{R_{n},\left\langle X_{n}\right\rangle\right\}$ with respect to $f_{n q}$ analytically. After simple algebra, the minimization condition gives the expression of the variational transformation parameter $f_{n q}$ as $f_{n q}=g_{n q} \mathcal{F}_{n q}$, where

$$
\mathcal{F}_{n q}=\frac{\omega_{q} \frac{\partial F_{u}}{\partial R_{n}}}{\omega_{q} \frac{\partial F_{u}}{\partial R_{n}}-\frac{1}{2} \frac{\partial F_{u}}{\partial\left\langle X_{n}\right\rangle} \operatorname{coth}\left(\beta \omega_{q} / 2\right)\left\langle X_{n}\right\rangle} .
$$

Since $\left\{R_{n},\left\langle X_{n}\right\rangle\right\}$ are also functions of $f_{n q}$, above equation must be solved self-consistently. After the minimization procedure, the optimal choice of $\bar{H}_{0}$ and $\bar{H}_{I}$ is obtained. In Sec. III, dissipative quantum transport formalism with variational polaron transformation is presented.

\section{DISSIPATIVE QUANTUM TRANSPORT THEORY WITH VARIATIONAL POLARON TRANSFORMATION}

Non-equilibrium Green's function (NEGF) formalism is employed to study the dissipative quantum transport. The key quantity in the NEGF method is the single-particle Green's function, which is given by

$$
\begin{aligned}
G_{m n}\left(\tau, \tau^{\prime}\right) & =-i\left\langle T_{c} c_{m}(\tau) c_{n}^{\dagger}\left(\tau^{\prime}\right)\right\rangle_{H} \\
& =-i\left\langle T_{c} c_{m}(\tau) X_{m}(\tau) c_{n}^{\dagger}\left(\tau^{\prime}\right) X_{n}^{\dagger}\left(\tau^{\prime}\right)\right\rangle_{\bar{H}},
\end{aligned}
$$

where $H$ and $\bar{H}$ are the Hamiltonians before and after the variational polaron transformation, respectively. $\tau$ and $\tau^{\prime}$ are the time variables defined on the Keldysh contour and $T_{c}$ is the contour time-ordering operator. Eq. (13) determines the dynamics of the coupled electrons and phonons. We employ the following approximation to decouple the electron and phonon dynamics:

$$
G_{m n}\left(\tau, \tau^{\prime}\right)=\bar{G}_{m n}\left(\tau, \tau^{\prime}\right) K_{m n}\left(\tau, \tau^{\prime}\right)
$$

where

$$
\begin{aligned}
\bar{G}_{m n}\left(\tau, \tau^{\prime}\right) & =-i\left\langle T_{c} c_{m}(\tau) c_{n}^{\dagger}\left(\tau^{\prime}\right)\right\rangle_{\bar{H}}, \\
K_{m n}\left(\tau, \tau^{\prime}\right) & =\left\langle T_{c} X_{m}(\tau) X_{n}^{\dagger}\left(\tau^{\prime}\right)\right\rangle_{\bar{H}} .
\end{aligned}
$$

The decoupling in Eq. (14) is similar to the concept of BornOppenheimer approximation. This approach is valid when the dynamics of phonon and electron have different time scales. But it should be noted that the decoupling approximation used in Eq. (14) has not been fully justified to the best of our knowledge.

With the decoupling approximation, the key quantity becomes $\bar{G}\left(\tau, \tau^{\prime}\right)$ which is associated with $\bar{H}$. As illustrated 
in Sec. II, the variational polaron transformed Hamiltonian can be divided into two parts: $\bar{H}_{0}$ and $\bar{H}_{I} . \bar{H}_{0}$ is mean-field part of the Hamiltonian, the quantum transport problem associated with $\bar{H}_{0}$ can be easily solved as demonstrated by previous publications, ${ }^{43}$ while the correction of $\bar{H}_{I}$ to the quantum transport can be taken into consideration by the perturbation theory because the variational polaron transformation ends up with small $\bar{H}_{I}$. First, zero-order Green's function $G_{0}\left(\tau, \tau^{\prime}\right)$ is obtained by using $\bar{H}_{0}$,

$$
\begin{aligned}
G_{0}\left(\tau, \tau^{\prime}\right)= & g_{0}\left(\tau, \tau^{\prime}\right)+\int d \tau_{1} \int d \tau_{2} g_{0}\left(\tau, \tau_{1}\right) \\
& \times\left[\sum_{\alpha} \bar{\Sigma}_{0 \alpha}\left(\tau_{1}, \tau_{2}\right)\right] G_{0}\left(\tau_{2}, \tau^{\prime}\right),
\end{aligned}
$$

where $g_{0}\left(\tau, \tau^{\prime}\right)$ is the electron Green's function in absence of system-lead coupling. The effect of system-lead coupling is represented by the self-energy,

$$
\begin{aligned}
\bar{\Sigma}_{0 \alpha, m n}\left(\tau, \tau^{\prime}\right) & =\sum_{k_{\alpha}} V_{k_{\alpha} m}^{*} V_{k_{\alpha} n} g_{k_{\alpha}}\left(\tau, \tau^{\prime}\right)\left\langle X_{m}\right\rangle\left\langle X_{n}\right\rangle \\
& \equiv \Sigma_{\alpha, m n}\left(\tau, \tau^{\prime}\right)\left\langle X_{m}\right\rangle\left\langle X_{n}\right\rangle,
\end{aligned}
$$

where $g_{k_{\alpha}}\left(\tau, \tau^{\prime}\right)$ is the surface Green's function of lead $\alpha$ and $\Sigma_{\alpha}\left(\tau, \tau^{\prime}\right)$ is the lead self-energy in absence of phonon. Next, $G\left(\tau, \tau^{\prime}\right)$ is derived at second-order expansion with respect to $\bar{H}_{I}$, starting from $G_{0}\left(\tau, \tau^{\prime}\right)$.

\section{A. Second-order perturbation theory}

Within the 2PT theory, the Green's function can be expressed as

$$
\begin{aligned}
\bar{G}\left(\tau, \tau^{\prime}\right)= & \bar{G}_{0}\left(\tau, \tau^{\prime}\right)+\int d \tau_{1} \int d \tau_{2} \bar{G}_{0}\left(\tau, \tau_{1}\right) \\
& \times \bar{\Sigma}_{I}\left(\tau_{1}, \tau_{2}\right) \bar{G}_{0}\left(\tau_{2}, \tau^{\prime}\right)
\end{aligned}
$$

where $\bar{\Sigma}_{I}\left(\tau, \tau^{\prime}\right)$ is the self-energy to Green's function at the second-order with respect to $\bar{H}_{I}$ and $\bar{G}_{0}\left(\tau, \tau^{\prime}\right)$ is the Green's function corresponding to $\bar{H}_{0}$. Since $\bar{H}_{I}$ contains two parts, $\bar{H}_{S \alpha}^{I}$ and $\bar{H}_{S B}, \bar{\Sigma}_{I}\left(\tau, \tau^{\prime}\right)$ is divided into two parts accordingly,

$$
\bar{\Sigma}_{I}\left(\tau, \tau^{\prime}\right)=\bar{\Sigma}_{e p}\left(\tau, \tau^{\prime}\right)+\sum_{\alpha} \bar{\Sigma}_{I \alpha}\left(\tau, \tau^{\prime}\right)
$$

Within the $2 \mathrm{PT}, \Sigma_{I}\left(\tau, \tau^{\prime}\right)$ is derived as

$$
\bar{\Sigma}_{I \alpha, m n}\left(\tau, \tau^{\prime}\right)=\Sigma_{\alpha, m n}\left(\tau, \tau^{\prime}\right)\left\langle T_{C} B_{n}\left(\tau^{\prime}\right) B_{m}^{\dagger}(\tau)\right\rangle_{\bar{H}_{0}} .
$$

The definition of $\bar{\Sigma}_{I \alpha}$ is very similar to Eq. (17) except the phonon renormalization factor $\left\langle X_{i}\right\rangle$ is replaced by the correlation function of $B_{i}$. According to the definition of $B_{i}$, the self-energy $\Sigma_{\alpha, I}$ can be rewritten as

$$
\begin{aligned}
\bar{\Sigma}_{I \alpha, m n}\left(\tau, \tau^{\prime}\right) & =\Sigma_{\alpha, m n}\left(\tau, \tau^{\prime}\right)\left[K_{n m}\left(\tau^{\prime}, \tau\right)-\left\langle X_{m}\right\rangle\left\langle X_{n}\right\rangle\right] \\
& =\Sigma_{\alpha, m n}\left(\tau, \tau^{\prime}\right) K_{n m}\left(\tau^{\prime}, \tau\right)-\bar{\Sigma}_{0 \alpha, m n}\left(\tau, \tau^{\prime}\right),
\end{aligned}
$$

where $\bar{\Sigma}_{0 \alpha}\left(\tau, \tau^{\prime}\right)$ is the phonon dressed self-energy, which is used to evaluate the zero-order Green's function $\bar{G}_{0}\left(\tau, \tau^{\prime}\right)$ as described previously.

The derivation of $\bar{\Sigma}_{e p}(\tau, \tau)$ is same as that in Ref. 6. The only difference is that the electron-phonon coupling constant $\left(g_{n q}\right)$ is replaced by the reduced one $\left(\bar{g}_{n q}\right)$. The expression of
$\bar{\Sigma}_{e p}\left(\tau, \tau^{\prime}\right)$ is

$$
\Sigma_{e p}\left(\tau, \tau^{\prime}\right)=\bar{g} D_{0}\left(\tau, \tau^{\prime}\right) \bar{G}_{0}\left(\tau, \tau^{\prime}\right) \bar{g},
$$

where $D_{0}\left(\tau, \tau^{\prime}\right)$ is the bare phonon Green's function.

In practical implementation, the Green's function and self-energies defined on the Keldysh contour are projected on real-time axis, resulting in equations for retarded/advanced and lesser/greater Green's functions and self-energies. In this work, we focuses on the steady state. In steady state, all quantities (two-time functions) depends only on the time difference instead of two times. Hence, Fourier transformation is employed to transfer the quantities from real-time domain to energy space. Since $\bar{H}_{0}$ is the mean-field part of the total Hamiltonian, the corresponding Green's functions can be easily evaluated as

$$
\begin{aligned}
& \bar{G}_{0}^{r}(E)=\left[E+i \eta-\bar{H}_{0}-\sum_{\alpha} \bar{\Sigma}_{0 \alpha}^{r}(E)\right]^{-1}, \\
& \bar{G}_{0}^{<}(E)=\bar{G}_{0}^{r}(E)\left[\sum_{\alpha} \bar{\Sigma}_{0 \alpha}^{<}(E)\right] \bar{G}_{0}^{a}(E),
\end{aligned}
$$

where $\eta \rightarrow 0^{+} . \bar{\Sigma}_{0 \alpha}^{r}$ and $\bar{\Sigma}_{0 \alpha}^{<}$are the phonon dressed retarded and lesser self-energies of lead $\alpha$, respectively, which are written as

$$
\begin{aligned}
\bar{\Sigma}_{0 \alpha, m n}^{r /<}(E) & =\sum_{k_{\alpha}} V_{k_{\alpha} m}^{*} V_{k_{\alpha} n} g_{k_{\alpha}}^{r /<}(E)\left\langle X_{m}\right\rangle\left\langle X_{n}\right\rangle \\
& =\Sigma_{\alpha, m n}^{r /<}(E)\left\langle X_{m}\right\rangle\left\langle X_{n}\right\rangle,
\end{aligned}
$$

where $\Sigma_{\alpha, m n}^{r /<}(E)$ is the lead self-energy in energy space in absence of phonon. Defining the unperturbed linewidth function as $\Gamma_{\alpha}(E)=-2 \mathfrak{J}\left[\Sigma_{\alpha}^{r}(E)\right]$, where $\mathfrak{I}$ denotes the imaginary part, the phonon dressed linewidth function becomes $\bar{\Gamma}_{\alpha, m n}(E)=\Gamma_{\alpha, m n}(E)\left\langle X_{m}\right\rangle\left\langle X_{n}\right\rangle$. The dressing factor indicates the localization of electron induced by the electron-phonon interaction. In term of linewidth function, the corresponding lesser self-energy can be rewritten as $\bar{\Sigma}_{0 \alpha}^{<}(E)=i f_{\alpha}(E) \bar{\Gamma}_{\alpha}(E)$ according to the fluctuation-dissipation law, where $f_{\alpha}(E)$ is the Fermi-Dirac distribution function of lead $\alpha$ with chemical potential $\mu_{\alpha}$.

After the zeroth-order Green's functions, $\bar{G}_{0}^{r /<}(E)$, are evaluated, the Green's functions with $\bar{H}_{I}$ are obtained through the $2 \mathrm{PT}$ theory as

$$
\begin{aligned}
\bar{G}^{r}(E) & =\bar{G}_{0}^{r}(E)+\bar{G}_{0}^{r}(E) \bar{\Sigma}_{I}^{r}(E) \bar{G}_{0}^{r}(E), \\
\bar{G}^{<}(E) & =\bar{G}_{0}^{<}(E)+\bar{G}_{0}^{r}(E) \bar{\Sigma}_{I}^{<}(E) \bar{G}_{0}^{a}(E),
\end{aligned}
$$

where $\bar{\Sigma}_{I}^{r /<}(E)$ is the self-energy to Green's function at the second-order with respect to $\bar{H}_{I}$. As shown previously, $\bar{\Sigma}_{I}^{r /<}(E)$ contains two parts, $\bar{\Sigma}_{I}^{r /<}(E)=\bar{\Sigma}_{e p}^{r /<}(E)+\sum_{\alpha} \bar{\Sigma}_{I \alpha}^{r /<}$ $(E)$. Retarded and lesser phonon self-energies within the 2PT $\operatorname{are}^{6,44,45}$

$$
\begin{aligned}
\bar{\Sigma}_{e p}^{<}(E)= & \sum_{q, \pm} \bar{g}_{q} N_{q}^{ \pm} \bar{G}_{0}^{<}\left(E \pm \omega_{q}\right) \bar{g}_{q}, \\
\bar{\Sigma}_{e p}^{r}(E)= & \sum_{q, \pm} \bar{g}_{q}\left[N_{q}^{\mp} \bar{G}_{0}^{r}\left(E \pm \omega_{q}\right) \pm \bar{G}_{0}^{<}\left(E \mp \omega_{q}\right)\right] \bar{g}_{q} \\
& +\bar{\Sigma}^{H},
\end{aligned}
$$

where $\bar{\Sigma}^{H}$ is the Hartree part of the retarded self-energy which represents phonon-induced energy shift. Its element is $\bar{\Sigma}_{m}^{H}=-i \sum_{n} \frac{2 \bar{g}_{m q} \bar{g}_{n q}}{\omega_{q}} \int \bar{G}_{n}^{<}(E) \frac{d E}{2 \pi} \cdot N_{q}^{ \pm}=N_{q}+\frac{1}{2} \pm \frac{1}{2} \cdot N_{q}$ is the 
phonon occupation number of mode $q$, which is determined by Bose-Einstein distribution function.

The derivation of $\Sigma_{I \alpha}^{r /<}(E)$ requires the knowledge of the displacement correlation function $K\left(\tau, \tau^{\prime}\right) . K\left(\tau, \tau^{\prime}\right)$ in principle depends on the phonon Green's function which is coupled with electronic Green's function via its selfenergy. ${ }^{27-29}$ Therefore, self-consistent calculation of phonon and electron Green's function is required. However, numerical implementation of self-consistent calculation is non-trivial and very computational demanding. In practice, we assume the phonon is in equilibrium and undressed by the electron in this work. The quality of the assumption is justified by the interplay of electron-phonon interaction and the dissipation of phonon's energy to bath. In the strong damping limit, phonon dissipates the extra energy got from electron quickly to the environment. Then phonon is close to equilibrium. In contrast, phonon is driven far from equilibrium if the damping is weak. The influence of electron to the phonon can be introduced through a phenomenological rate equation including the renormalization, damping, and heating effect. ${ }^{46-48}$ With the assumption that phonon is in the equilibrium and undressed by electron, the displacement correlation function can be rewritten in a simple form. ${ }^{25,27,42}$ The lesser projection of displacement correlation function is expressed as

$$
\begin{aligned}
K_{m n}^{<}\left(t, t^{\prime}\right)= & \left\langle X_{n}^{\dagger}\left(t^{\prime}\right) X_{m}(t)\right\rangle \\
= & \prod_{q=1}\left\{e ^ { - \frac { \lambda _ { m q } ^ { 2 } + \lambda _ { n q } ^ { 2 } } { 2 } ( 2 N _ { q } + 1 ) } \operatorname { e x p } \left\{\lambda_{m q} \lambda_{n q}\right.\right. \\
& \left.\times\left[N_{q} e^{-i \omega_{q}\left(t-t^{\prime}\right)}+\left(N_{q}+1\right) e^{i \omega_{q}\left(t-t^{\prime}\right)}\right]\right\},
\end{aligned}
$$

where $N_{q}$ is the occupation number for the $q$ th phonon mode. $K_{m n}^{<}\left(t, t^{\prime}\right)$ can be decomposed as

$$
K_{m n}^{<}\left(t, t^{\prime}\right)=\sum_{p} L_{m n}^{p} e^{i p^{\mathrm{T}} \boldsymbol{\omega}\left(t-t^{\prime}\right)}
$$

where both $\boldsymbol{p}$ and $\boldsymbol{\omega}$ are the row vectors, $\boldsymbol{p}^{\mathrm{T}} \boldsymbol{\omega}=\sum_{q} p_{q} \omega_{q}$. And $L_{m n}^{p}=L_{m n}^{p_{1}} L_{m n}^{p_{2}} \ldots L_{m n}^{p_{M}}$, where $L_{m n}^{p_{q}}$ is modified Bessel function

$$
L_{m n}^{p_{q}}=e^{-\frac{\lambda_{i q}^{2}+\lambda_{j q}^{2}}{2}\left(2 N_{q}+1\right)} e^{p_{q} \omega_{q} \beta / 2} I_{p_{q}}\left[2 \lambda_{m q} \lambda_{n q} \sqrt{N_{q}\left(N_{q}+1\right)}\right],
$$

and $I_{p_{q}}$ is the $p_{q}$ th order Bessel function.

The greater projection of displacement correlation function is $K_{m n}^{>}\left(t, t^{\prime}\right)=\left\langle X_{m}(t) X_{n}^{\dagger}\left(t^{\prime}\right)\right\rangle=\left[K_{m n}^{<}\left(t, t^{\prime}\right)\right]^{\dagger}$. It can be verified that $K^{<}\left(t, t^{\prime}\right) \simeq K^{>}\left(t, t^{\prime}\right)$ in the high-temperature limit since $N_{q} \simeq N_{q}+1$ in this limit. Actually, there are previous works adopted $K^{<}\left(t, t^{\prime}\right) \simeq K^{>}\left(t, t^{\prime}\right)$ as an approximation in low temperature, which is regarded as neglecting the Fermi sea. $23-26,49$

With $K^{\lessgtr}\left(t, t^{\prime}\right)$ known, the lesser (greater) self-energy in energy space can be obtained from the Fourier transformation of $\Sigma_{I \alpha}^{\lessgtr}\left(t, t^{\prime}\right)$,

$$
\bar{\Sigma}_{I \alpha, m n}^{\lessgtr}(E)=\sum_{p} L_{n m}^{p} \Sigma_{\alpha, m n}^{\lessgtr}\left(E \mp \boldsymbol{p}^{T} \omega\right)-\bar{\Sigma}_{0 \alpha, m n}^{\lessgtr}(E) .
$$

\section{B. Observable of interest}

The steady-state current through the junction can be expressed in Meir-Wingreen formula ${ }^{43}$

$$
I_{\alpha}=\frac{2 e}{\hbar} \int \frac{d E}{2 \pi} T_{\alpha}(E),
$$

where the factor 2 accounts for the spin degeneracy and $T_{\alpha}$ is the transmission coefficient through lead $\alpha$, which is expressed in terms of Green's functions and self-energies within NEGF formalism,

$$
T_{\alpha}(E)=\operatorname{Tr}\left[\Sigma_{\alpha}^{<}(E) G^{>}(E)-\Sigma_{\alpha}^{>}(E) G^{<}(E)\right] .
$$

According to Eq. (14), lesser/greater projection of Green's functions is $G_{m n}^{\lessgtr}\left(t, t^{\prime}\right)=\bar{G}_{m n}^{\lessgtr}\left(t, t^{\prime}\right) K_{m n}^{\lessgtr}\left(t, t^{\prime}\right)$. Utilizing the expansion of $K_{m n}^{\lessgtr}\left(t, t^{\prime}\right)$, Fourier transformation of $G^{\lessgtr}\left(t, t^{\prime}\right)$ gives the Green's functions in energy space as

$$
G_{m n}^{\lessgtr}(E)=\sum_{p} L_{m n}^{p} \bar{G}_{m n}^{\lessgtr}\left(E \mp \boldsymbol{p}^{T} \omega\right) .
$$

Hence, transmission coefficient and current can be obtained through substituting Eq. (31) into Eq. (30). With lesser/greater Green's function known, the spectral can be expressed as $A(E)=i\left[G^{>}(E)-G^{<}(E)\right]$. Thus, density of states (DOS) can be obtained by tracing spectral function. In short, the computational procedures are briefly summarized as follows: (1) bare Green's functions $\bar{G}_{0}^{r, s}(E)$ in variational polaron picture are calculated; (2) Green's functions $\bar{G}^{r, s}(E)$ at secondorder with respect to $\bar{H}_{I}$ are evaluated; (3) Green's functions in the $H$ picture are obtained through Eq. (31); (4) physical quantities, such as DOS and currents, are obtained from the Green's functions and self-energies.

\section{NUMERICAL EXAMPLES}

The variational polaron theory for dissipative quantum transport is employed to study the transport through a monoatomic chain. The system consists of a single site. The two leads are modeled by semi-infinite linear chains as illustrated in Fig. 1. The onsite energy of the system is denoted by $\epsilon_{S}$. The onsite energy and hopping element of leads are $\epsilon$ and $t$, respectively. The system-lead coupling constant is denoted by $V_{\alpha}$. In this work, it is assumed that the system is coupled to the two leads equally, i.e., $V_{L}=V_{R}=v$. The linewidth function of the semi-infinite leads is $\Gamma_{\alpha}=2 V_{\alpha}^{2} / t$ within the Wide-Band-Limit (WBL) approximation. ${ }^{50}$

In this work, the system-lead coupling is set to be equal to the hopping in the leads, i.e., $v=t$. The onsite energy is set to be $0 \mathrm{eV}$ and hopping is set as $t=0.1 \mathrm{eV}$. And the Fermi energy of the leads is set as $0 \mathrm{eV}$, which corresponds to half-filled on each site. Temperature is set as $T=300 \mathrm{~K}$. In this work, only

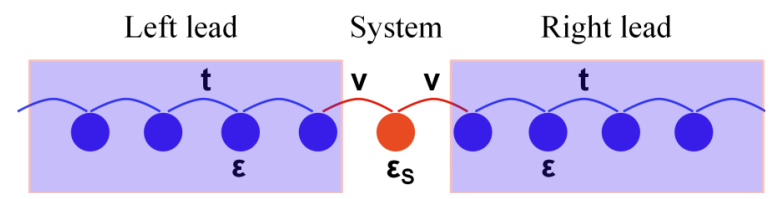

FIG. 1. Illustration of a single site model coupled to two leads. It is assumed that the system coupled to the two leads equally, which are denoted by $v$. The onsite energy and hopping element of leads are $\epsilon$ and $t$, respectively. 


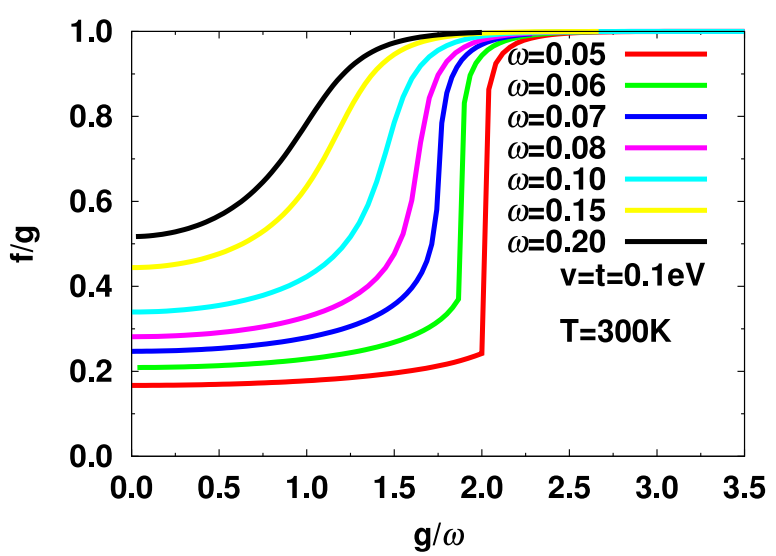

FIG. 2. Optimal value $f$ of variational polaron transformation against different electron-phonon coupling constant $g$. The system-lead coupling and hopping in leads are $v=t=0.1 \mathrm{eV}, T=300 \mathrm{~K}$.

one phonon mode is considered to be coupled to the system for simplicity. However, the current method is general and is applicable to multiple phonon modes. In fact, early works on spinboson model usually considered multiple phonon modes..$^{33,34}$

The optimal value $f$ of variational polaron transformation is searched for different phonon frequency and electronphonon coupling strength. The phonon frequency is chosen in three different regimes: $\omega>t, \omega=t$, and $\omega<t$. The optimal value $f$ of variational polaron transformation for different electron-phonon coupling constant $g$ is shown in Fig. 2. As it is expected, different electron-phonon coupling strength corresponds to different optimal value of $f$ in the variational transformation. Besides, $f$ increases monotonously with electron-phonon coupling strength $g$. When $g \rightarrow 0$, $\mathrm{f} / \mathrm{g}$ ratio approaches a small value which is affected by the phonon energy. In the $g \rightarrow 0$ limit, $f / g$ ratio is small, which approaches no polaron transformation limit. While in the strong electron-phonon coupling limit $(g \rightarrow \infty), f / g$ ratio approaches to 1 , which corresponds to full polaron transformation. In the intermediate regime, variational polaron transformation ends up with $f / g<1$, which denotes the partial polaron transformation.

It is also noticed from Fig. 2 that there is a sharp transition of $f$ in a certain regime of $g$ if $\omega<t$. Larger $\frac{t}{\omega}$ results in the steeper transition. Finally, $f$ shows discontinuity against $\frac{g}{\omega}$. This discontinuity appears due to different local minimum becomes the global minimum at certain parameter regime. Taking $\omega=0.05 \mathrm{eV}$ as example, the free energies against $f / g$ ratio with electron-phonon coupling strength around $0.1 \mathrm{eV}$ are plotted in Fig. 3. As it shown in Fig. 3, two local minimums start to emerge at $g \approx 0.1 \mathrm{eV}$. The local minimums of smaller and larger $f / g$ become the global minimum for $g=0.098 \mathrm{eV}$ and $g=0.102 \mathrm{eV}$, respectively. The discontinuity of $f$ may cause unphysical phase transition from delocalized to localized regime. ${ }^{34,51,52}$ For multiple site systems, the free energy can be more complex and more local minimums may emerge; thus, there is larger possibility of discontinuity. However, even the discontinuity appears, the variational transformation is still likely to work better than the untransformed scheme or polaron transformation. Moreover, the discontinuity of $f$ may be removed by employing certain ansatz. For instance, a ground state ansatz has been proposed recently to get rid of

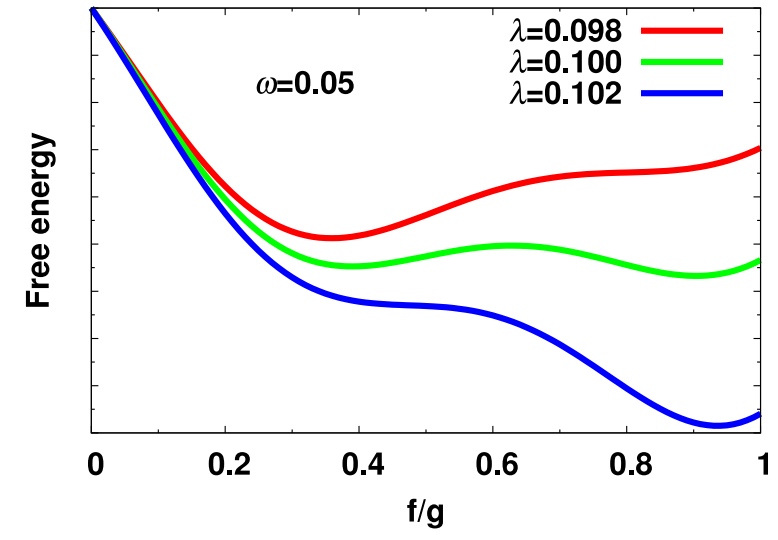

FIG. 3. Free energy as a function of variational transformation parameter $f$ for different electron-phonon coupling strength $g$. The phonon energy is $0.05 \mathrm{eV}$. When $g$ is around $0.10 \sim 0.102 \mathrm{eV}$, different local minimum may become the global minimum, resulting in abrupt transition of $f$.

the discontinuity in the variational polaron. ${ }^{52,53}$ Similar ansatz may be proposed to remove the discontinuity problem in the quantum transport system, which will be explored in further development of the method.

After the variational polaron transformation parameter is found, the quantum transport properties of the system, such as DOS and current-voltage characteristics, at different electron-phonon coupling regimes can be studied by the method described in Sec. III. Here, three sets of parameters are studied, ranging from weak to strong electron-phonon coupling regime. The parameters as well as optimal factor of variational polaron transformation are summarized in Table I. Models A, B, and C correspond to the weak, moderate, and strong coupling regimes, respectively. The optimal values of variational polaron transformation are $0.17,0.54$, and 0.90 . Here, the $g / \omega$ ratio is set as a constant for the three models. As shown in Fig. 2, large $g / \omega$ ratio $(g / \omega \gg 1)$ represents the system in polaron regime while small $g / \omega$ ratio $(g / \omega \ll 1)$ represents the system in weak or moderate coupling regime. Hence, $g / \omega=0.8$ is chosen to make sure that the system varies from the weak to strong coupling by tuning the electronphonon coupling constant.

In presence of electron-phonon coupling, electronic structure of the system is affected at different levels depending on the electron-phonon coupling strength. Consequently, DOS of the system is affected, and such effects include energy shift of peak, broadening of the spectral, and emergence of phonon side-bands. Fig. 4 plots the DOS of the system with different electron-phonon coupling strength.

(a) In model A, the electron-phonon coupling strength is small. Phonon has limited effects on the electronic structure of the system. The phonon-induced energy shift is about $50 \mathrm{meV}$. The DOS of variational polaron transformation is close to that of 2PT. Moreover, the DOS of both variational

TABLE I. Parameters of the models.

\begin{tabular}{lccc}
\hline \hline Model & $\omega(\mathrm{eV})$ & $g(\mathrm{eV})$ & $f / g$ \\
\hline A & 0.05 & 0.04 & 0.17 \\
B & 0.15 & 0.12 & 0.54 \\
C & 0.40 & 0.32 & 0.90 \\
\hline \hline
\end{tabular}




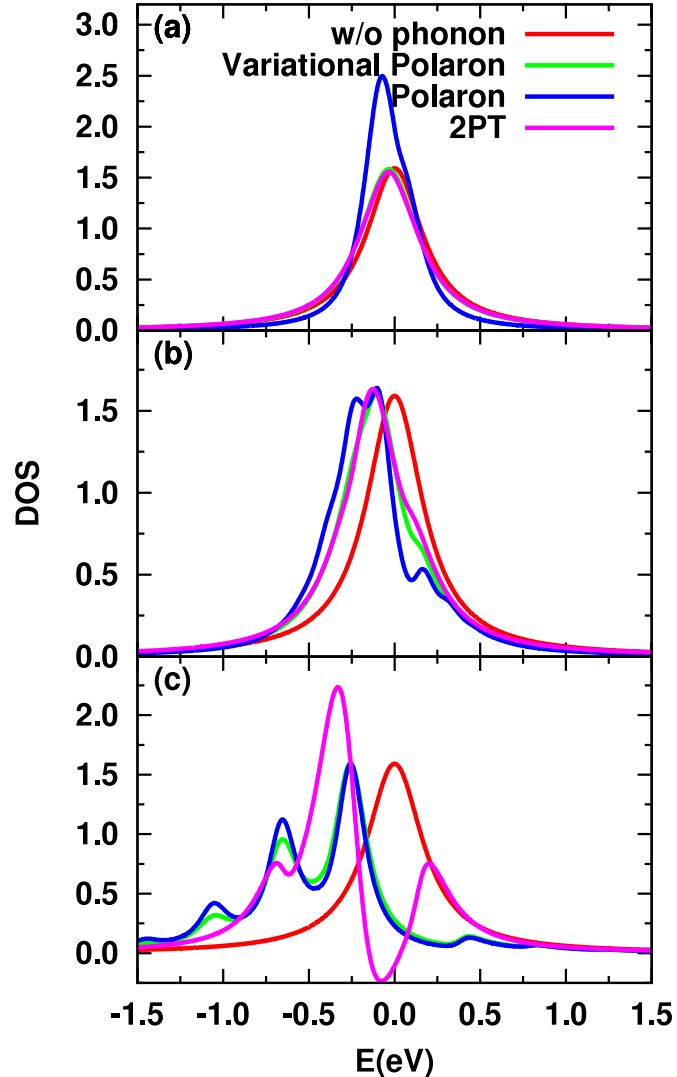

FIG. 4. DOS of the system with different electron-phonon coupling constant: (a) $\omega=0.05 \mathrm{eV}$ and $g=0.04 \mathrm{eV}$. The electron-phonon interaction is in the weak coupling regime. DOS of variational polaron is same as that of $2 \mathrm{PT}$, while polaron theory overestimates the phonon side-bands. (b) $\omega=0.15 \mathrm{eV}$ and $g=0.12 \mathrm{eV}$. In this moderate electron-phonon coupling regime, 2PT starts to fail and polaron still overestimates the phonon side-bands. (c) $\omega$ $=0.40 \mathrm{eV}$ and $g=0.32 \mathrm{eV}$. The system is in the strong coupling regime. Variational polaron theory gives similar results as polaron theory. While 2PT is not applicable in this regime, which gives unphysical DOS. See text for other parameters.

polaron theory and 2PT is almost the same as that of noninteracting system except the phonon-induced shift of the peak. No obvious phonon side-bands are observed in the DOS of variational polaron theory and 2PT. However, polaron theory overestimates the energy shift and phonon side-bands, resulting in stronger localization effect. Consequently, main peak of DOS is sharpened and phonon side-band appears as shown in Fig. 4(a).

(b) In the moderate coupling regime, phonon has larger effect on the electronic structure of the system and phonon side-bands start to appear due to the increased phonon-induced electron localization. In this regime, the DOS of variational polaron theory starts to deviate from that of $2 \mathrm{PT}$ as shown by Fig. 4(b). Due to the principle of 2PT, only two phonon side-bands can be observed in the DOS. However, higher order corrections become more and more significant in the moderate coupling regime. As a result, $2 \mathrm{PT}$ fails to describe the renormalization effect induced by phonon. Besides the phonon side-bands, the energy shift due to electron-phonon interaction is underestimated slightly by $2 \mathrm{PT}$. In contrast, higher order phonon side-bands can be evaluated by both polaron theory and variational polaron theory. However, polaron theory overestimates the phonon dressed system-lead coupling compared with variational polaron theory. And the phonon side-bands are much more significant in the DOS. Our results shows that variational polaron theory correctly describes the renormalization effect. The DOS of variational polaron lies between those of 2PT and polaron.

(c) In the strong coupling limit, phonon induces pronounced side-bands effect and polaron is formed. Consequently, the variational polaron transformation approaches the polaron theory. As shown in Fig. 4(c), DOS of variational polaron theory is almost the same as that of polaron theory since the optimal value of $f / g$ is 0.9 , which is close to 1 , i.e., variational polaron transformation approaches polaron transformation. In contrast, $2 \mathrm{PT}$ greatly underestimates the phonon side-bands, phonon-induced energy shift, and even gives unphysical negative DOS. The pronounced phonon side-bands indicate that electron is localized by the phonon. The phonon side-bands are also responsible for the phonon blockade in the current-voltage characteristics, which is represented by steps in the current-voltage characteristics or peaks in the differential conductance. ${ }^{24-27}$

The corresponding current-voltage curves of the system with different electron-phonon coupling strengths are shown in Fig. 5. In the weak coupling regime, current calculated by

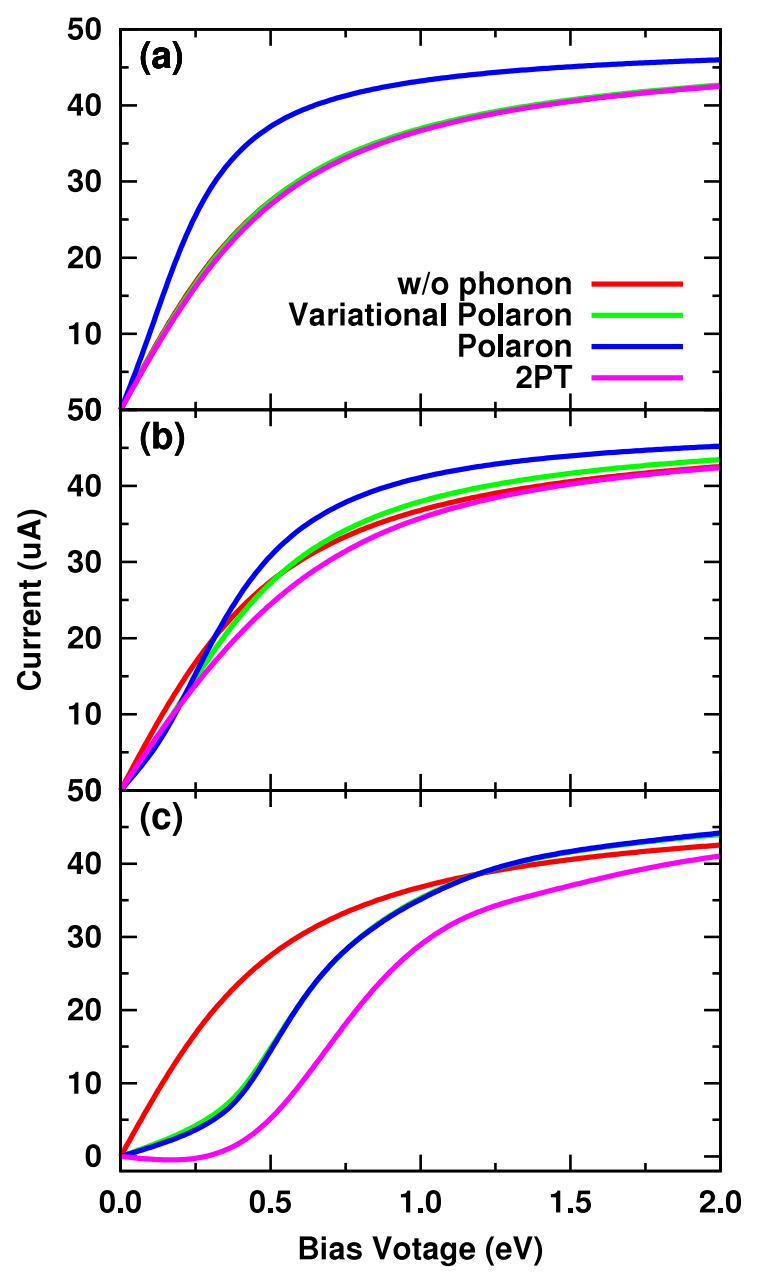

FIG. 5. Current of the system with different electron-phonon coupling constant. The chemical potentials are set as $\mu_{L}=-\Delta V / 2$ and $\mu_{R}=\Delta V / 2$, where $\Delta V$ is the bias voltage. (a) $\omega=0.05 \mathrm{eV}$ and $g=0.04 \mathrm{eV}$; (b) $\omega=0.15 \mathrm{eV}$ and $g=0.12 \mathrm{eV}$; (c) $\omega=0.40 \mathrm{eV}$ and $g=0.32 \mathrm{eV}$. Other parameters are same as those of Fig. 4. 
the variational polaron approaches agrees well with that of $2 \mathrm{PT}$, while polaron theory overestimates the current since it overestimates the DOS. In the strong coupling regime, the current predicted by variational polaron is consistent with polaron theory, while 2PT underestimates the current (it even gives negative current at low bias) due the unphysical negative DOS as shown in Fig. 4(c). The comparison of current-voltage characteristics confirms that the variational polaron theory is applicable to a wide parameter space of electron-phonon coupling strength.

\section{SUMMARY}

In this work, a dissipative quantum transport theory with electron-phonon coupling in a wide parameter space is developed by employing the variational polaron transformation. Variational polaron transformation provides an optimal set of parameters for the unitary transformation, which is determined by the minimization of the Feynman-Bogoliubov upper bound of free energy. After the minimization, the variational polaron transformation leads to an optimal meanfield and a perturbation Hamiltonian.

The mean-field part Hamiltonian, $\bar{H}_{0}$, contains the phonon-induced energy renormalization and phonon dressed system-lead coupling. Thanks to the variational polaron transformation, the many-body effect of electron-phonon interaction is transformed into the small interacting part of the Hamiltonian $\left(\bar{H}_{I}\right)$, which validates the $2 \mathrm{PT}$ treatment. Upon the variational transformation, a quantum transport theory with electron-phonon interaction in a wide parameter space is established within NEGF formalism. Following the quantum transport theory of non-interaction system, the mean-field part is treated exactly. $\bar{H}_{I}$ is taken into account through the selfenergies which are in second-order of $\bar{H}_{I}$.

Numerical examples on an atomic chain demonstrate the validity of the variational transformation. The optimal value of variational transformation increases monotonously with electron-phonon coupling strength, which connects the 2PT in weak coupling regime and polaron theory in strong coupling limit. Comparison of DOS and current-voltage characteristics in different parameter regimes indicates the applicability of the variational polaron transformation: (a) In weak coupling limit, polaron theory overestimates the phononinduced energy shift and side-bands; (b) in the strong coupling limit, 2PT underestimates the renormalization effect induced by phonon and even gives unphysical DOS; (c) variational polaron transformation naturally connects the $2 \mathrm{PT}$ in the weak coupling limit and polaron theory in the strong coupling limit by making use of the optimal transformation. More numerical benchmark comparison with numerically exact methods, such as QUAPI and MCTDH, will be considered in the future works.

The variational polaron theory for dissipative quantum transport is expected to work well over arbitrary parameter space. However, it works best for fast phonon, i.e., phonon frequency is not smaller than the system frequency $(\omega>t)$. For slow phonon, discontinuity emerges in the search of optimal transformation. The emergence of discontinuity limits the application of the method in certain parameter space. However, it is expected that the discontinuity only exists in a limited parameter space. Moreover, the discontinuity problem of finding optimal variational transformation can be solved by employing appropriate ansatz, ${ }^{52,53}$ which can be the future improvement of the method.

\section{ACKNOWLEDGMENTS}

Y. Zhang thanks Jianshu Cao, Weitao Yang, E.K.U. Gross, and Garnet Chan for helpful discussions. The support from the Hong Kong Research Grant Council (Contract Nos. HKU7009/09P, 7009/12P, 7007/11P, and HKUST9/CRF/11G (GHC)), the University Grant Council (Contract No. AoE/P04/08 (GHC)), National Natural Science Foundation of China (NSFC Nos. 21322306 (CYY) and 21273186 (GHC, CYY)), and National Basic Research Program of China (No. 2014CB921402 (CYY)) is gratefully acknowledged.

${ }^{1}$ M. Kumar, R. Avriller, A. L. Yeyati, and J. M. van Ruitenbeek, Phys. Rev. Lett. 108, 146602 (2012).

${ }^{2}$ N. Agraiit, C. Untiedt, G. Rubio-Bollinger, and S. Vieira, Phys. Rev. Lett. 88, 216803 (2002)

${ }^{3}$ M. Paulsson, T. Frederiksen, H. Ueba, N. Lorente, and M. Brandbyge, Phys. Rev. Lett. 100, 226604 (2008).

${ }^{4}$ Y. Dubi and M. Di Ventra, Rev. Mod. Phys. 83, 131 (2011).

${ }^{5}$ G. Romano, A. Gagliardi, A. Pecchia, and A. Di Carlo, Phys. Rev. B 81, 115438 (2010).

${ }^{6}$ Y. Zhang, C. Y. Yam, and G. H. Chen, J. Chem. Phys. 138, 164121 (2013).

${ }^{7}$ C. Y. Yam, Y. Mo, F. Wang, X. Li, G. H. Chen, X. Zheng, Y. Matsuda, J. Tahir-Kheli, and W. A. Goddard III, Nanotechnology 19, 495203 (2008).

${ }^{8}$ T. Brixner, J. Stenger, H. M. Vaswani, M. Cho, R. E. Blankenship, and G. R. Fleming, Nature 434, 625 (2005).

${ }^{9}$ J. Wu, F. Liu, Y. Shen, J. Cao, and R. J. Silbey, New J. Phys. 12, 105012 (2010).

${ }^{10}$ M. Cho, H. M. Vaswani, T. Brixner, J. Stenger, and G. R. Fleming, J. Phys. Chem. B 109, 10542 (2005).

${ }^{11}$ M. Galperin, M. A. Ratner, and A. Nitzan, Nano Lett. 5, 125 (2005).

${ }^{12}$ H. Park, J. Park, A. K. L. Lim, E. H. Anderson, A. P. Alivisatos, and P. L. McEuen, Nature 407, 57 (2000).

${ }^{13}$ Y. Tanimura, J. Phys. Soc. Jpn. 75, 082001 (2006).

${ }^{14}$ A. Ishizaki and Y. Tanimura, J. Phys. Soc. Jpn. 74, 3131 (2005).

${ }^{15}$ N. Makri and D. E. Makarov, J. Chem. Phys. 102, 4600 (1995); 102, 4611 (1995).

${ }^{16}$ M. Beck, A. Jäckle, G. Worth, and H.-D. Meyer, Phys. Rep. 324, 1 (2000),

${ }^{17}$ M. Thoss, H. Wang, and W. H. Miller, J. Chem. Phys. 115, 2991 (2001).

${ }^{18}$ H.-D. Meyer, U. Manthe, and L. Cederbaum, Chem. Phys. Lett. 165, 73 (1990).

${ }^{19}$ D. P. S. McCutcheon and A. Nazir, Phys. Rev. B 83, 165101 (2011); New J. Phys. 12, 113042 (2010).

${ }^{20}$ A. Nazir, Phys. Rev. Lett. 103, 146404 (2009).

${ }^{21}$ S. Jang, J. Chem. Phys. 131, 164101 (2009); 135, 034105 (2011).

${ }^{22}$ S. Jang, Y.-C. Cheng, D. R. Reichman, and J. D. Eaves, J. Chem. Phys. 129, 101104 (2008)

${ }^{23}$ N. S. Wingreen, K. W. Jacobsen, and J. W. Wilkins, Phys. Rev. B 40, 11834 (1989).

${ }^{24}$ U. Lundin and R. H. McKenzie, Phys. Rev. B 66, 075303 (2002).

${ }^{25}$ Z.-Z. Chen, R. Lü, and B.-F. Zhu, Phys. Rev. B 71, 165324 (2005).

${ }^{26}$ J.-X. Zhu and A. V. Balatsky, Phys. Rev. B 67, 165326 (2003).

${ }^{27}$ M. Galperin, A. Nitzan, and M. A. Ratner, Phys. Rev. B 73, 045314 (2006).

${ }^{28}$ R. Härtle, C. Benesch, and M. Thoss, Phys. Rev. B 77, 205314 (2008); R. Härtle, M. Butzin, and M. Thoss, ibid. 87, 085422 (2013).

${ }^{29}$ Y. Zhang, C. Y. Yam, and G. H. Chen, J. Chem. Phys. 142, 164101 (2015).

${ }^{30}$ D. P. S. McCutcheon, N. S. Dattani, E. M. Gauger, B. W. Lovett, and A. Nazir, Phys. Rev. B 84, 081305 (2011).

${ }^{31}$ F. A. Pollock, D. P. S. McCutcheon, B. W. Lovett, E. M. Gauger, and A. Nazir, New J. Phys. 15, 075018 (2013).

${ }^{32}$ D. P. S. McCutcheon and A. Nazir, J. Chem. Phys. 135, 114501 (2011).

${ }^{33}$ R. Silbey and R. A. Harris, J. Chem. Phys. 80, 2615 (1984); R. A. Harris and R. Silbey, ibid. 83, 1069 (1985).

${ }^{34}$ C. K. Lee, J. Moix, and J. Cao, J. Chem. Phys. 136, 204120 (2012). 
${ }^{35}$ X. Zheng, G. H. Chen, Y. Mo, S. Koo, H. Tian, C. Y. Yam, and Y. Yan, J. Chem. Phys. 133, 114101 (2010).

${ }^{36}$ X. Zheng, F. Wang, C. Y. Yam, Y. Mo, and G. H. Chen, Phys. Rev. B 75, 195127 (2007).

${ }^{37}$ X. Zheng, C. Y. Yam, F. Wang, and G. H. Chen, Phys. Chem. Chem. Phys. 13, 14358 (2011).

${ }^{38}$ Y. Zhang, S. Chen, and G. H. Chen, Phys. Rev. B 87, 085110 (2013).

${ }^{39}$ H. Xie, F. Jiang, H. Tian, X. Zheng, Y. Kwok, S. Chen, C. Y. Yam, Y. Yan, and G. H. Chen, J. Chem. Phys. 137, 044113 (2012).

${ }^{40}$ C. Y. Yam, S. Yokojima, and G. H. Chen, J. Chem. Phys. 119, 8794 (2003).

${ }^{41}$ C. Y. Yam, X. Zheng, G. H. Chen, Y. Wang, T. Frauenheim, and T. A. Niehaus, Phys. Rev. B 83, 245448 (2011).

${ }^{42}$ G. D. Mahan, Many-Particle Physics, 3rd ed. (Kluwer Academic, New York, 2000).

${ }^{43}$ A.-P. Jauho, N. S. Wingreen, and Y. Meir, Phys. Rev. B 50, 5528 (1994).

${ }^{44}$ Y. Zhang, L. Meng, C. Y. Yam, and G. H. Chen, J. Phys. Chem. Lett. 5, 1272 (2014).
${ }^{45}$ M. Bescond, C. Li, H. Mera, N. Cavassilas, and M. Lannoo, J. Appl. Phys. 114, 153712 (2013).

${ }^{46}$ M. Paulsson, T. Frederiksen, and M. Brandbyge, Phys. Rev. B 72, 201101 (2005).

${ }^{47}$ T. Frederiksen, M. Brandbyge, N. Lorente, and A.-P. Jauho, Phys. Rev. Lett. 93, 256601 (2004).

${ }^{48}$ K. Kaasbjerg, T. Novotný, and A. Nitzan, Phys. Rev. B 88, 201405 (2013).

${ }^{49} \mathrm{H}$. Haug and A.-P. Jauho, Quantum Kinetics in Transport and Optics of Semiconductors (Springer, 2008).

${ }^{50} \mathrm{E}$. Scheer, Molecular Electronics: An Introduction to Theory and Experiment (World Scientific, 2010).

${ }^{51}$ D. Yarkony and R. Silbey, J. Chem. Phys. 65, 1042 (1976).

${ }^{52}$ A. Nazir, D. P. S. McCutcheon, and A. W. Chin, Phys. Rev. B 85, 224301 (2012).

${ }^{53}$ S. Bera, A. Nazir, A. W. Chin, H. U. Baranger, and S. Florens, Phys. Rev. B 90, 075110 (2014). 